\title{
Can Cultural Competency Reduce Racial And Ethnic Health Disparities? A Review And Conceptual Model
}

\author{
Cindy Brach \\ Irene Fraserirector \\ Agency for Healthcare Research and Quality
}

\begin{abstract}
This article develops a conceptual model of cultural competency's potential to reduce racial and ethnic health disparities, using the cultural competency and disparities literature to lay the foundation for the model and inform assessments of its validity. The authors identify nine major cultural competency techniques: interpreter services, recruitment and retention policies, training, coordinating with traditional healers, use of community health workers, culturally competent health promotion, including family/community members, immersion into another culture, and administrative and organizational accommodations. The conceptual model shows how these techniques could theoretically improve the ability of heal th systems and their clinicians to deliver appropriate services to diverse populations, thereby improving outcomes and reducing disparities. The authors conclude that while there is substantial research evidence to suggest that cultural competency should in fact work, health systems have little evidence about which cultural competency techniques are effective and less evidence on when and how to implement them properly.
\end{abstract}

With minority Americans expected to comprise more than 40 percent of the U.S. population by 2035 and 47 percent by 2050 (U.S. Bureau of the Census 1996), addressing their health needs has become an increasingly visible public policy goal (Agency for Health Care Policy and Research 1999; U.S. Department of Health and Human Services [DHHS] Office for Civil Rights 1998; U.S.

Thanks are due to Marc Chow and Carolyn Wang for their research assistance and Maggie Rutherford for her editing assistance. Thanks are also due to the many reviewers whose incisive comments helped strengthen the article. These include Carolyn Clancy, Julia Puebla Fortier, Cathy Hoffman, Marsha Lillie-Blanton, Nicole Lurie, Nathan Stinson, and the staff at the Office of

Medical Care Research and Review, Vol. 57 Supplement 1, (November 2000) 181-217

(C) 2000 Sage Publications, Inc. 
DHHS 1999; U.S. DHHS Office of the Secretary 1999). Making sure that the health care provided to this diverse population takes account of their linguistic and cultural needs constitutes a major challenge for health systems and policy makers.

Arthur Kleinman's seminal Annals of Internal Medicine article (Kleinman, Eisenberg, and Good 1978) articulated the importance of culture in health care. Culture, defined as the "integrated pattern of human behavior that includes thoughts, communications, actions, customs, beliefs, values and institutions of a racial, ethnic, religious or social group" (Cross et al. 1989) is relevant to everyone's health care. However, its importance is heightened for minority patients who receive health care from systems that are largely organized by and staffed with majority group members. Examples of negative health consequences that could result from ignoring culture include missed opportunities for screening because of lack of familiarity with the prevalence of conditions among certain minority groups; failure to take into account differing responses to medication; lack of knowledge about traditional remedies, leading to harmful drug interactions; and diagnostic errors resulting from miscommunication (Lavizzo-Mourey and Mackenzie 1996; Lawson 1996; Moffic and Kinzie 1996). A growing body of federal and state laws, regulations, and standards seeks to guarantee that health systems respond to these diverse linguistic and cultural needs by becoming "culturally competent" ( ${ }^{*}$ ADD TO REFERENCES LIST* Sec. 601, Civil Rights Act of 1964; 78 Stat. 252; 42 U.S.C. 2000d; Chang and Fortier 1998; Fortier and Shaw-Taylor 1999; Kennedy, Stubblefield-Tave, and Smith 1999; Perkins et al. 1998; Perkins and Vera 1998; *ADD TO REFERENCES* 63 Fed. Reg, 52021 et seq. 29 September 1998; U.S. DHHS Office of the Secretary 1999; Woloshin et al. 1995).

Every organization and author define cultural competency somewhat differently (e.g., Adams 1995; Association of Asian Pacific Community Health Organizations 1996; Association of State and Territorial Hospital Officials 1994; Orlandi 1995; Tirado 1996; U.S. DHHS Goal 6 Work Group *wORKGROUP1 WORD?SEE REFERENCE* 1998). However, most definitions are variants of one developed by mental health researchers more than a decade ago, who defined cultural competence as "a set of congruent behaviors, attitudes, and policies that come together in a system, agency or amongst professionals and enables that system, agency or those professionals to work effectively in cross-cultural situations" (Cross et al. 1989).

Minority Health, Beau Stubblefield-Tave, and two anonymous reviewers. The views expressed in this article are those of the authors and do not necessarily reflect the views of the Agency for Healthcare Research and Quality. 
Because this article considers cultural competency in the context of reducing racial and ethnic health disparities, we limit our use of the term to racial and ethnic minority groups, although the term cultural competency has been used to include other groups (such as women, the elderly, gays and lesbians, people with disabilities, and religious minorities). We do, however, include linguistic competence when we speak of cultural competency. Predicated on theories that language and culture affect health care beliefs, choices, and treatment (Ashing-Giwa 1999; Flores and Vega 1998; Gordon 1995; Gropper 1998; Kleinman, Eisenberg, and Good 1978; Lipton et al. 1998; Pachter 1994; Torres 1998), the idea of cultural competency is an explicit statement that one-size-fits-all health care cannot meet the needs of an increasingly diverse American population. Cultural competency goes beyond cultural awareness or sensitivity. It includes not only possession of cultural knowledge and respect for different cultural perspectives but also having skills and being able to use them effectively in cross-cultural situations (Cross et al. 1989; Orlandi 1995; Tirado 1996). Taken one step further, the term cultural competency has been used to refer to an ongoing commitment or institutionalization of appropriate practice and policies for diverse populations (Denboba et al. 1998; Tervalon and Murray-Garcia 1998). The concept of cultural competency is also presented as a continuum, in recognition that individuals and institutions can vary in the effectiveness of their responses to cultural diversity (Andrulis, Delbanco, and Shaw-Taylor 1999; Cross et al. 1989).

Some advocate for cultural competency as a matter of social justice. According to this perspective, the high value we as a society place on informed consent, choice of providers, and equity creates an entitlement to cultural competency regardless of its impact on outcomes (Kennedy, Stubblefield-Tave, and Smith 1999; Richardson 1999). Cultural competency is therefore a mechanism to ensure the observance of consumer rights, such as the right to respect and nondiscrimination enunciated in the Consumer Bill of Rights and Responsibilities (President's Advisory Commission on Consumer Protection and Quality in the Health Care Industry 1997).

Cultural competency, however, could also be a mechanism to change the health outcomes of minority Americans. A large body of literature has documented significant racial and ethnic disparities in health care and health outcomes, with minority Americans generally receiving less health care and suffering worse health (Baquet and Commiskey 1999; Collins, Hall, and Neuhaus 1999; Ferguson et al. 1998; Gaston et al. 1993; Mayberry et al. 1999; Sheifer, Escarce, and Schulman 2000; U.S. DHHS 1999). The question addressed in this article is as follows: Could cultural competency reduce racial and ethnic health disparities? 


\section{NEW CONTRIBUTION}

We conducted a review of both the cultural competency and disparity literature using Medline and HealthStar, concentrating on articles published within the past 10 years. More than 40 search terms (e.g., cultural competency, disparity, minority, race, ethnicity, language barriers, access) were used in various combinations. In addition, we reviewed bibliographies that had been compiled by various sources, such as a workgroup of the DHHS Secretary's Quality Initiative and the DHHS Office of Minority Health Resource Center. Finally, we contacted knowledgeable sources in this field and uncovered unpublished or nonindexed literature as well. We discovered that the literature on cultural competency has, by and large, not linked cultural competency activities with the outcomes that could be expected to follow from them. We also found the literature on racial and ethnic disparities weak on identifying the sources of disparities, and almost no attention has been paid to techniques for reducing them. Researchers have generally focused on rigorously documenting disparities and offered only speculative explanations for their findings.

This article brings together the two bodies of literature to explore whether cultural competency has the potential to reduce disparities. It begins by identifying the major cultural competency techniques described in the literature. Then it draws on the literature on cultural competency and health care disparities to provide a conceptual model and evidence of ways in which cultural competency techniques could affect the processes-and, therefore, outcomesof care for racial and ethnic minorities.

\section{CULTURAL COMPETENCY TECHNIQUES}

The need for cultural competency is frequently discussed on the level of the patient-clinician interaction. (The term clinician as used in this article encompasses physicians, nurses, and other health professionals.) However, most health care is now delivered by clinicians who are part of groups or systems. These clinicians will become culturally competent only with the support and/or encouragement of the health systems in which they participate. Furthermore, cultural competency must be addressed by health systems if it is to become institutionalized. Much of the cultural competency literature discusses the importance of cultural awareness, knowledge, attitudes, and skills but does not describe how a health system is supposed to become culturally competent. While the literature overall identifies a wide array of cultural competency techniques, most articles discuss only a single approach, making it difficult for health systems to possess an overview of the options available to 
them. We have therefore described the techniques most frequently discussed in the cultural competency literature, clustering them into nine categories.

1. Interpreter services. Providing foreign language or American Sign Language interpreter services is one obvious and common way to improve communication among persons who speak different languages and come from different cultures. There are numerous approaches to interpretation, including on-site professional interpreters; ad hoc interpreters (e.g., staff pulled away from other duties to interpret, friends and family members, strangers from the waiting room); and simultaneous remote interpretations, using earphones and microphones with off-site professional interpreters (Hornberger et al. 1996; Riddick 1998). Professional interpreters can be part-time or fulltime employees, acquired through an agency, or hired on a freelance basis (Riddick 1998).

2. Recruitment and retention. Minority staff, because of their shared cultural beliefs and common language, may improve communication, create a more welcoming environment, and structure health systems to better reflect the needs of minority communities (Cooper-Patrick et al. 1999; Nickens 1992). Techniques for recruiting and retaining minority group members in health systems include (1) setting up minority residency or fellowship programs, (2) hiring minority search firms, (3) adapting personnel policy to create a comfortable and welcoming work place for minority group members, (4) mentoring of minority employees by senior executives, (5) subcontracting with minority health providers, (6) tying executive compensation to steps taken to match hiring to community needs, (7) expanding on traditional affirmative action programs aimed at attracting employees who match the race and ethnicity of the patient populations, (8) establishing a set of principles for respectful treatment of all people, (9) reviewing fairness of human resource practices and compensation of all staff, and (10) tracking staff satisfaction by racial and ethnic groups (Chang and Fortier 1998; B. R. Williams 1997).

3. Training. Cultural competency training programs aim to increase cultural awareness, knowledge, and skills, leading to changes in staff (both clinical and administrative) behavior and patient-staff interactions. Training provides a way to ameliorate problems stemming from the cultural mismatches that result whenever patient and staff do not share a common subculture and mutual understanding of each other's health beliefs. Persons may be culturally competent in serving members similar to themselves but not serving others. The diversity even within racial or ethnic groups and the complexity of associated subcultures make cultural competency training appropriate for all health system staff, including members of minority groups. Training can be 
part of undergraduate or graduate medical or other professional school education, an orientation process for new staff or network members, or part of in-service training programs. Cultural competency training can be a separate activity, either a regularly occurring activity or a discrete one-time occurrence. Cultural competency training also can be achieved by infusion, integrating a multicultural perspective throughout a curriculum or training activities. Training can be targeted to increasing knowledge about specific minority groups and/or can focus on the process of interacting with minority group members. Training also can be focused on a specific task, such as working with interpreters. In lieu of or in addition to in-person training, handbooks and other educational materials can be distributed.

4. Coordinating with traditional healers. Many minority Americans use traditional healers while they are seeking biomedical care (Elder, Gillcrist, and Minz 1997; Kim and Kwok 1998; Ma 1999; Marbella et al. 1998; Skaer et al. 1996; Pang 1989). Clinicians need to coordinate with these healers as they would with any other care provider whom a patient is seeing to ensure continuity of care and avoid complications due to incompatible therapies. In addition, presenting patient education and treatment regimens in a conceptual framework concordant with cultural beliefs and traditional health practices may increase the chances that patients will concur with and adhere to behavioral and treatment recommendations.

5. Use of community health workers. Internationally and across the United States, members of minority communities are used to reach out to other community members as well as to provide direct services such as health education and primary care (e.g., E. A. Baker et al. 1997; Earp and Flax 1999; Jackson and Parks 1997; Rodney et al. 1998; Sherer 1994). Because they are known and respected by the community, these liaisons reportedly serve as guides to the health system. They bring in individuals who had not previously sought care, provide cultural linkages, overcome distrust, and contribute to clinician-patient communication, increasing the likelihood of patient follow-up and providing cost-effective health services to isolated communities that have traditionally lacked access (Goicoechea-Balbona 1997; Riddick 1998; Witmer et al. 1995; Zablocki 1998).

6. Culturally competent health promotion. Health promotion seeks to encourage good health through healthy behaviors and risk reduction, early detection and treatment, and proper care of chronic or acute diseases (Kok, van den Borne, and Mullen 1997). Health promotion can take several forms. Clinicians can use screening tools and conduct brief interventions. Public information campaigns can be conducted by health systems or in the community. 
In an attempt to make health-promotion efforts more culturally competent, culture-specific attitudes and values have been incorporated into messages and materials.

7. Including family and/or community members. While patient autonomy has become a core principle of health care in the United States, some minority groups believe that family members should be involved in health care decision making. For example, one study found that Korean and Mexican Americans were more likely than European or African Americans to hold a family-centered model, preferring that family members-not patients-be told about terminal conditions and make treatment decisions (Blackhall et al. 1995). Involving families-and, in hierarchical societies, even community leaders-may be crucial in obtaining consent for and adherence to treatment (Fadiman 1997).

8. Immersion into another culture. Members of one cultural group have been reported to develop sensitivity and skills working with another culture by immersing themselves in that culture (Kavanagh et al. 1999; St. Clair and McKenry 1999). It is reported that immersion enables participants to overcome their ethnocentrism, increase their cultural awareness, and integrate cultural beliefs into health care practices (St. Clair and McKenry 1999).

9. Administrative and organizational accommodations. A variety of administrative and organizational decisions related to clinic locations, hours of operation, network membership, physical environments, and written materials also can affect access to and utilization of health care. Health systems can make sure that providers are located near enrollees, especially if public transportation is not readily available. Health systems may even decide to go on the road to reach their population (Lane 1998). They can also make sure they are not consigning minority patients to a restricted pool of clinicians and, in particular, that minority patients are provided equal access to high-quality clinicians whose expertise is in great demand. Systems can also alter physical environments to make them more welcoming to minority group members. They can make sure that linguistic competency extends beyond the clinical encounter to the appointment desk, advice lines, and membership and other written materials. Many of these techniques have been combined in sites targeting specific minority populations (e.g., Chinese or Hispanic health centers).

\section{CULTURAL COMPETENCY AND HEALTH DISPARITIES}

Health systems might wish to adopt one or more of the cultural competency techniques described above for several reasons. They might want to 
increase their market share of growing minority populations (Torres 1998) and certainly will want to comply with growing federal and state regulations calling for culturally competent care. One important reason to adopt them, however, could be as a way to reduce racial and ethnic health disparities.

The literature reveals a consistent gap between majority and minority populations in terms of outcomes of health care. Black women are more likely than white women to die from breast cancer, despite having a lower incidence of the disease (Collins, Hall, and Neuhaus 1999). Infant mortality rates are 2.5 times greater for African Americans and 1.5 times greater for Native Americans than for white Americans (U.S. DHHS 1998a). Influenza death rates are higher for African Americans and American Indians/Native Alaskans than they are for white Americans (Grantmakers in Health 1998). Mortality for colorectal cancer is highest for African Americans, followed by Native Alaskans, and then Hawaiians (Baquet and Commiskey 1999). Might cultural competency techniques make some inroad to reduce these disparities?

Racial and ethnic disparities have many diverse causes. Low socioeconomic status (SES) is certainly a major cause. Minority Americans are disproportionately represented among the poor, the unemployed, and the undereducated, and low SES is correlated with poorer access to health care services and poorer health outcomes (Lillie-Blanton and Laveist 1996). But even minority Americans who are not socioeconomically disadvantaged have systematically different health experiences from nonminority Americans (Ford and Cooper 1995; Gornick et al. 1996; Mayberry et al. 1999; Kington and Smith 1997; D. R. Williams 1999; Nickens 1995).

Furthermore, minority Americans have different experiences in the health care system, even when they have similar medical conditions and insurance coverage. For example, disparities have been documented within health systems that provide equal financial benefits to all covered individuals-such as the Veterans Health Administration, Medicare, and single health plans (Ayanian et al. 1993; Carlisle, Leake, and Shapiro 1997; Conigliaro et al. 2000; Goldberg et al. 1992; Oddone et al. 1999; Peterson et al. 1994; Robbins, Whittemore, and Van Den Eeden 1998). Since financial barriers should not be a factor in these cases, researchers have concluded that the health care delivery system, for whatever reasons, must be doing an inferior job in meeting the needs of racial and ethnic minorities than in meeting the needs of the nonminority population.

In the next section, we explore how one cultural competency techniqueinterpreter services-might theoretically reduce these disparities in care and outcomes. We also review the research evidence that lays the foundation for the model and informs assessments of its validity. 


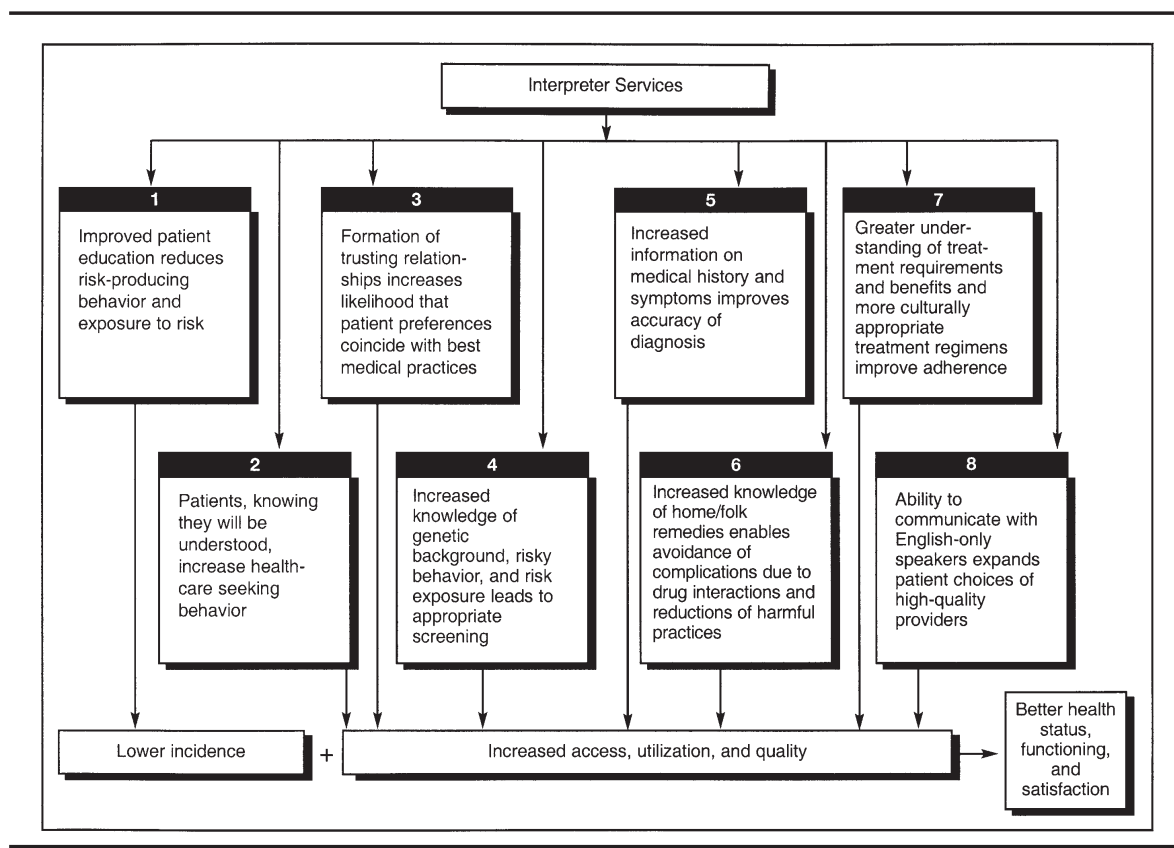

FIGURE 1 Conceptual Model of How Interpreter Services Could Reduce Health Disparities

\section{INTERPRETER SERVICES: CONCEPTUAL MODEL AND RESEARCH EVIDENCE}

Any of the nine cultural competency techniques described above could, in theory, reduce disparities by reducing errors and improving care. Using the example of interpreter services, Figure 1 provides a conceptual model of how this process might work.

Language differences present a problem for 21 percent of minority Americans receiving health care (Commonwealth Fund 1995). Research shows that language barriers apparently have a negative impact on utilization, satisfaction, and possibly adherence. This research serves as a basis for the inference that interpreter services could have an impact on disparities. Analyses have linked language barriers or limited English proficiency (LEP) with fewer physician visits and reduced receipt of preventive services, even after controlling for such factors as literacy, health status, health insurance, regular source of care, and economic indicators (Derose and D. W. Baker 2000; Solis et al. 1990; Stein, Fox, and Murata 1991; Wagner and Guendelman 2000; Woloshin et al. 
1997). Researchers have documented lower rates of satisfaction among LEP patients, even when compared with patients of the same ethnicity who have good English skills (Carrasquillo et al. 1999; David and Rhee 1998; Morales et al. 1999).

Findings on associations between language barriers and adherence rates have been slightly more equivocal. While some studies have found that patients who did not speak the same language as their clinicians were more likely to miss appointments or drop out of treatment (Manson 1988; Takeuchi, Uehara, and Maramba 1999), others have found that language barriers did not affect missed appointment or dropout rates (Flaskerud and Liu 1991; Sarver and D. W. Baker 2000). The mixed findings on the relationship between language barriers and adherence mirror the literature's failure to find a clear relationship between general clinician-patient communication and adherence (Kaplan, Greenfield, and Ware 1989).

Figure 1 provides a schematic representation of how interpreter services might reduce linguistic barriers and therefore reduce disparities. Each box in Figure 1 describes how the provision of interpreter services could change the health care experience for the LEP patient in such a way that outcomes would be improved. The model presented in this section is conceptual, describing the theoretical links between cultural competency and reductions in disparities.

In the first box, we theorize that having an interpreter will facilitate more successful patient education, resulting in reduced behaviors that constitute risk factors for disease and exposure to risk, thereby lowering disease ${ }^{1}$ incidence. For example, through interpreters, clinicians can counsel patients to stop smoking and suggest quitting strategies, which is a successful smokingcessation approach (U.S. DHHS 1998b).

In the second and third boxes, we theorize that having an interpreter could change patients' health care-seeking behavior and health care preferences. Research suggests that patterns of health care-seeking behavior vary from one cultural group to another and that different groups exhibit distinct health care preferences, such as disdaining technological procedures or favoring home settings over medical facilities (Ayanian et al. 1993; Maynard et al. 1986; Morrow-Howell et al. 1996; Oddone et al. 1998; Sheifer, Escarce, and Schulman 2000; Wenneker and Epstein 1989). Reasons for these differences include mistrust of white people and institutions, previous negative experiences with culturally insensitive or discriminatory practices or other problems with health care, higher utilization of alternative medicine (which may divert people from obtaining conventional health care), apprehensiveness about unfamiliar diagnostic techniques and treatment, lack of familiarity with disease processes and treatment options, and culturally determined health beliefs (Ashing-Giwa 1999; Ayanian et al. 1993; Commonwealth Fund 1995; Facione 1999; Ferguson 
et al. 1998; Flores and Vega 1998; Frederick Schneiders Research 1999; Gordon 1995; Kaiser Family Foundation 1999; Lannin et al. 1998; Pachter 1994; Sheifer, Escarce, and Schulman 2000; Uba 1992; Whittle et al. 1997). The result can be delayed treatment or no treatment at all (Peterson et al. 1994; Weissman et al. 1991). Having interpreters allows clinicians and LEP patients to communicate more clearly about their belief models of illness and treatment options. Patients who feel better understood and form more trusting relationships with their clinicians might seek out health care more frequently and might be more likely to adopt preferences for clinically indicated treatment.

The fourth box depicts how interpreter services could lead to more appropriate testing and screening - and fewer missed opportunities for early detection and treatment-by increasing clinicians' knowledge of patients' =genetic background, behavior, and risk exposure. Interpreter services could also reduce the amount of unnecessary testing LEP patients undergo. The presence of language barriers is associated with higher rates of diagnostic tests, an association that researchers speculate is due to physicians' attempts to compensate for difficulties in communication by ordering additional tests (David and Rhee 1998; Hampers et al. 1999).

More accurate medical histories as well as clearer descriptions of symptoms could lead to fewer diagnostic errors being made, as described in the fifth box. For example, some Latino parents have reported that failure of medical staff to speak Spanish led to adverse health consequences for their children, including poor medical care, misdiagnoses, and inappropriate prescriptions (Flores et al. 1998).

The sixth box shows that the use of interpreters could help clinicians discover the home/folk remedies patients are taking and thereby avoid dangerous interactions between biomedical treatment and home/folk remedies (Cameron 1999; Flores and Vega 1998; Pachter 1994). Some home/folk remedies can actually be harmful; uncovering their use and discussing them through interpretation could lead to a reduction in those practices (Pachter 1994).

In the seventh box, we theorize that provision of interpreter services could result in greater adherence to treatment regimens. Failure to adhere to prescribed treatment is a generally acknowledged problem in the health care system, with only about half of patients on long-term regimens continuing to adhere (Sackett and Snow 1979). Racial and ethnic disparities have been found among patients who make a treatment choice but fail to follow through on all its requirements (Eraker, Kirscht, and Becker 1984). Disparities have been found in missed appointment rates (which, e.g., have been linked to lower breast cancer survival rates) and omitted or discontinued medication (Howard, Penchansky, and Brown 1998; Hurley et al. 1998; Manson 1988). While researchers are just beginning to explore the reasons for these 
differences in adherence rates, studies show that folk beliefs, health beliefs and attitudes, social isolation and support, and beliefs about physiological differences among groups all affect adherence (Cameron 1996; Gropper 1998; Heurtin-Roberts and Reisin 1992). Enhanced communication through an interpreter could bring to light these beliefs that influence patients to discontinue treatment. They also could permit the clinician to share other perspectives and provide additional information about the requirements and benefits of treatment. Interpreter services could contribute to the joint development of a treatment plan that is more likely to be adhered to because it is informed by the cultural context in which the patient operates.

Finally, the eighth box shows the theory that the presence of interpreter services expands patients' choices and access to high-quality clinicians. Minority communities, regardless of their income, face a documented shortage of physicians, and this shortage can translate into poorer access for minority patients despite overall system capacity (Goldberg et al. 1992; Komaromy et al. 1996). Two factors combine to create such shortages. Physicians tend to care for a large percentage of patients from their own race or ethnic group (Komaromy et al. 1996), and minority group members are underrepresented in the medical profession (Libby, Zhou, and Kindig 1997). Providing interpreter services could help bridge the gap and encourage LEP patients to patronize health systems that do not have staff who speak their language.

In sum, each of the plausible consequences of interpreter services described in the eight boxes of the model has an impact on disease incidence, access, utilization, and / or quality. Reductions in risk result in less illness and fewer injuries. Increases in health care-seeking behavior and trust lead to higher access and utilization and possibly to more appropriate treatment. More appropriate screening, reductions in diagnostic errors, avoidance of drug interactions, and increasing adherence are all improvements in quality that flow from interpreter services. Finally, expanded choice of providers for patients increases access. The bottom row of boxes in Figure 1 shows that these reductions in incidence and increases in access, utilization, and quality combine to produce a healthier, better functioning, and more satisfied population.

The literature provides some evidence to support the linkages posed in the model. One study of why Vietnamese refugees in the United States do not optimally use health care services suggests that the sheer presence of interpreters could have a positive impact on utilization. The study revealed a paramount concern with not having an interpreter in health care facilities, and study participants expressed a willingness to seek health care more frequently if interpreters were available ( $\mathrm{D}^{\prime}$ Avanzo 1992). Another study showed that Spanish-speaking emergency department (ED) patients who spoke through an interpreter were more satisfied than those who said an interpreter should 
have been used (D. W. Baker, Hayes, and Fortier 1998). Enguidanos and Rosen (1997) found that interpreters eliminated disparities in adherence; there was no significant difference in the number of missed follow-up appointments from ED referrals between Spanish-speaking patients who used professional interpreters and English-speaking patients. Tocher and Larson (1998) found that with the provision of professional interpreters, outcomes for non-English-speaking diabetic patients (including standardized glycohemoglobin and other laboratory results as well as complication rates) were as good as outcomes for those who spoke English.

These studies did not compare patients who received interpreter services with those who did not. However, they collectively demonstrate that patients who have interpreter services do not suffer from the disparities documented in other studies comparing English-speaking and LEP patients. They provide some evidence that the provision of professional interpreters can increase utilization and satisfaction, reduce nonadherence, and eliminate disparities in quality of care and outcomes.

The other potential associations identified in Figure 1 are congruent with past research but need much more careful study. For example, research has documented that the quality of physician-patient communication affects outcomes (Kaplan, Greenfield, and Ware 1989; Ong et al. 1995; Stewart et al. 2000; Stewart 1995). In particular, a review of the literature found that the quality of communication while taking medical histories and during the discussion of the management plan influenced patients' health outcomes (Stewart 1995). It is not a large leap to extend the findings of this set of research to postulate that the provision of interpreter services would have many of the effects theorized in the model, but clearly we need more systematic research to explicitly assess the impact of interpreter services on health education, trust and health care preferences, diagnostic accuracy and errors, complications from interactions with home/folk remedies, and access to a wider array of health care clinicians.

Another question requiring further research concerns the different impact of various types of interpreter services. Although the services of professional interpreters are obviously more costly than those of ad hoc interpreters (Xuo and Fagan 1999), the practice of using ad hoc interpreters presents problems such as confidentiality, imperfect knowledge of medical terminology, and potential harmful impact on family relationships (Riddick 1998). Furthermore, the research referenced above linking language barriers to lower satisfaction held even when ad hoc translators were present. In the study by Carrasquillo and colleagues (1999), non-English speakers were less satisfied than English speakers with ED care even though ad hoc interpreters were used most of the time (H. Burstin, director, Center for Primary Care Research, Agency for Healthcare Research and Quality, personal communication, 17 
May 2000). Similarly, Hispanics who chiefly used ad hoc interpreters were less satisfied than Hispanics with good English skills or Hispanics who spoke with clinicians in Spanish (D. W. Baker, Hayes, and Fortier 1998; David and Rhee 1998). Spanish-speaking patients attended by native Spanish-speaking physicians also reported better well-being and functioning than did patients who communicated through ad hoc interpreters (Pérez-Stable, Napoles-Springer, and Miramontes 1997; E. J. Pérez-Stable, personal communication, 3 June 1999). Using ad hoc interpreters, however, should not be ruled out if there are no alternatives. In the D. W. Baker, Hayes, and Fortier (1998) study, those with mostly ad hoc interpreters were more satisfied than those who said an interpreter should have been used but was not.

\section{BROADENING THE MODEL}

This conceptual model of how interpreter services could reduce disparities can be broadened to include any of the eight other cultural competency techniques. Figure 2 illustrates this expansion of the model. Cultural competency techniques potentially could change both clinician and patient behavior by improving communication, increasing trust, creating a greater knowledge of the differences among racial and ethnic groups in epidemiology and treatment efficacy, and expanding understanding of patients' cultural behaviors and environment. The behavioral changes, in turn, could lead to the provision of more appropriate services: prevention and screening activities undertaken with full knowledge of risk factors, better informed diagnoses, treatment options formulated and presented in cultural contexts, and patient education on treatment regimens culturally tailored to improve the likelihood of adherence. When appropriate services are provided, good outcomes-such as higher levels of health status, increased functioning, and improved satisfaction-could then follow. The following sections draw on the evidence to suggest how five of the other cultural competency techniques shown in Figure 2 could reduce disparities: recruitment and retention of minority staff, training, coordinating with traditional healers, use of community health workers, and culturally competent health promotion. The analysis of these five techniques, along with the interpreter services technique discussed above, demonstrates how the theoretical promise of the three remaining techniques-techniques discussed less frequently in the literature-would be appraised.

\section{RECRUITMENT AND RETENTION OF MINORITY STAFF}

Provision of interpreter services does not appear to be a perfect substitute for language and cultural concordance, suggesting that there are advantages 


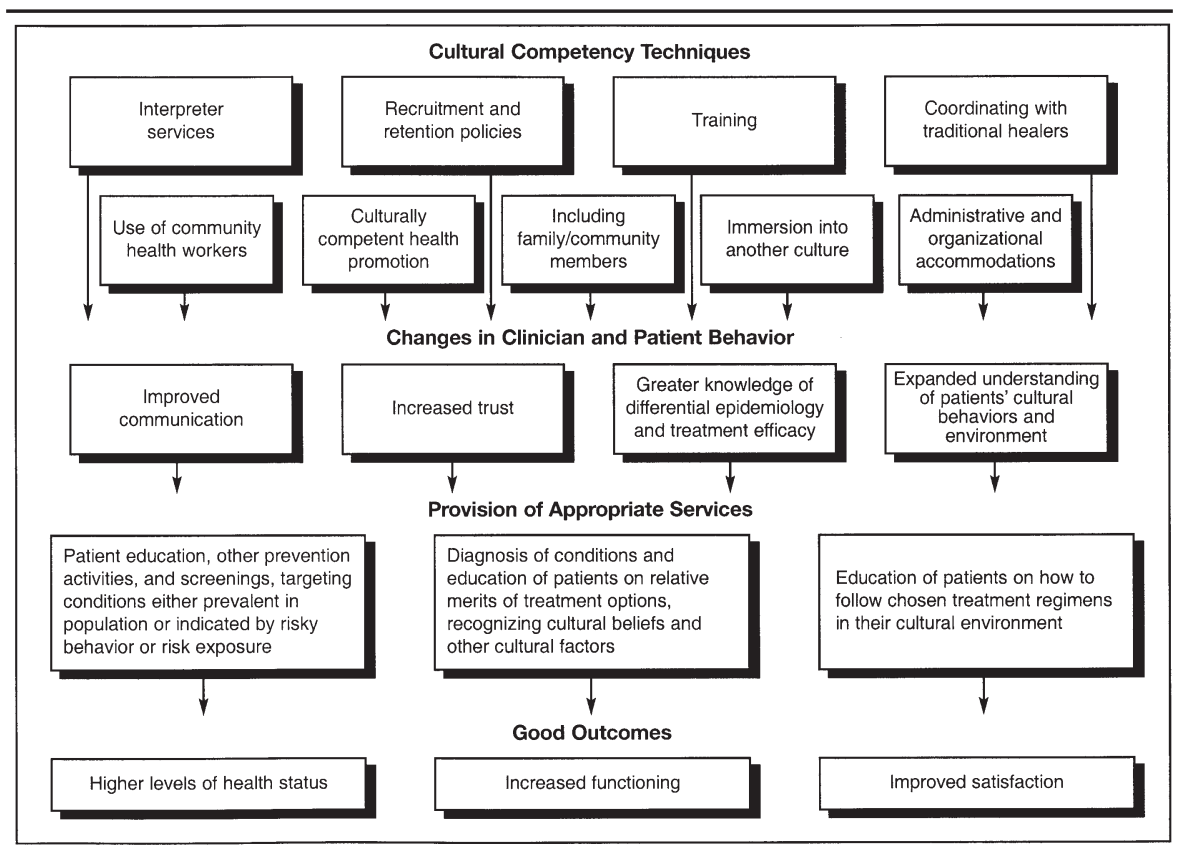

FIGURE 2 Conceptual Model of How Nine Cultural Competency Techniques Could Reduce Health Disparities

to health systems' recruiting and retaining staff who reflect the demographics of the patient population. Even with interpreters, Spanish-speaking patients make fewer comments than do English-speaking patients, are more likely to have their comments ignored by English-speaking physicians (Rivadeneyra et al. 2000), have worse recall of information communicated by their English-speaking physician, and ask fewer questions (Seijo, Gomez, and Freidenberg 1991). Lay interpreters can also introduce distortions that can lead to misevaluation of patients' mental health status (Marcos 1979).

There is also some evidence that rapport, quality of communication, and understanding of prescribed medications suffer when clinicians try to speak a language other than their own native language (Erzinger 1991; Shapiro and Saltzer 1981). This finding may be due as much to miscommunication stemming from different cultural perspectives as to lack of language proficiency. In one study where language differences were not involved, clinicians and patients communicated more effectively with each other when they belonged to the same racial group and therefore were more likely to be culturally similar. Cooper-Patrick and colleagues (1999) found that white and African Ameri- 
can patients who saw physicians of the same race rated the physician's decision-making style as more participatory and were more satisfied than were patients who saw physicians of a different race.

The superior communication that results when there is a language match between clinicians and patients could be expected to produce superior outcomes. The literature does indeed show a relationship between communication and adherence and outcomes (Ong et al. 1995; Stewart 1995; Stewart et al. 2000), although this connection has not been specifically demonstrated for language concordance. ${ }^{2}$

The rationale for racial and ethnic matches extends beyond just clinical personnel. Nickens (1992), while recognizing the lack of rigorous data, notes that there is much anecdotal evidence that "the presence of minority health professionals in health care settings increases the 'user friendliness' experienced by minority populations and hence accessibility." Nickens continues to observe that minority staff are likely to "organize the delivery system in ways more congruent with the needs of a minority population".

A final reason for health systems to recruit and retain minority staff is that minority staff might be less likely to practice racial or ethnic discrimination. Discrimination, either subconscious or overt, is believed to be a factor in some documented treatment differences, although confounding factors have made it difficult to prove conclusively (Ayanian et al. 1993; Egbert and Rothman 1977). However, two recent studies explicitly investigated whether physician biases influenced referral decisions by using videotaped actors who played the part of patients presenting with the same set of symptoms. In the first of these studies, Schulman et al. (1999) found that black women were less likely to be referred for cardiac catheterization than were white men and women or black men. While this study did not examine whether minority physicians are less likely than the overall group of physicians to exhibit biases in their referral decisions, a second study by many of the same investigators did. In this second study (Rathore et al. 2000), medical students encountering a black woman and white man with identical symptoms of definite angina perceived a lower quality of life for the black woman yet were less likely to assign her a diagnosis of definite angina. Students also thought the black woman was less likely to obtain follow-up care. Minority medical students did not share the same biases as their nonminority counterparts and assessed the black woman and white man similarly. The idea that minority group members might be less likely to discriminate was also suggested by a recent study of Peniston and colleagues (2000). Researchers found no significant differences in revascularization rates between black and white patients at a veterans' hospital where predominantly black physicians cared for predominantly black patients. This study's contrast to several other studies' findings of differential 
rates in settings not dominated by minority physicians suggests that racial and ethnic concordance has the potential to remedy disparities resulting from discrimination (East and Peterson 2000).

\section{TRAINING}

Training is another remedy for overcoming language and cultural barriers. Cultural competency training is designed to meet one or more of several goals: (1) to enhance self-awareness of attitudes toward minority group members, (2) to increase knowledge about minority populations (e.g., differential epidemiology, health behaviors, and medication response; cultural beliefs and practices; attitudes toward the health care system and health care-seeking behavior); and (3) to improve specific skills such as communication (D'Andrea, Daniels, and Heck 1991). The need for some sort of intervention is underscored by the findings of a recent study, which demonstrated that physicians perceive minority patients more negatively than white patients and noted that such attitudes have been shown to influence physician behavior (van Ryn and Burke 2000).

Some evaluation research has been conducted on the capacity of training to alter such attitudes, chiefly through the intermediary process of changing trainees' awareness and knowledge base. D'Andrea, Daniels, and Heck (1991) found that participants in multicultural counseling training perceived themselves as being more aware, knowledgeable, and skillful compared to both pretraining reports and controls. A study by Culhane-Pera and colleagues (1997) reported similar results from their evaluation of a multicultural curriculum for family practice residents. In their study, trainees' self-assessed increases in knowledge, cross-cultural communication skills, and cultural competency were corroborated by faculty assessments. Another report of a method for educating residents, a block rotation that integrates family systems theory, family-oriented skills, and sociocultural awareness, stated that residents completing evaluations 1 year after graduation indicated that these skills proved useful to them in practice (Marvel, Grow, and Morphew 1993).

While these studies largely rely on self-perceptions that could prove to be inaccurate, they are supported by the findings of the literature in general that training can be successful in changing physician behavior (Joos et al. 1996). Since communication skills and other aspects of physician behavior have been linked to patient adherence, outcomes, and satisfaction (Stewart 1995; Stewart et al. 2000), training could yield improvements in these areas. Relying on this chain of reasoning, interest and activity in cultural competency training have been heightened. There has been an increase in the prevalence of multicultural curricula in family practice residencies (Culhane-Pera et al. 2000). 
Eighty-seven percent of medical schools have courses in which cultural issues are presented in one or more of the lectures, and 8 percent of schools have separate courses specifically addressing cultural issues, although this level of undergraduate medical education on cultural sensitivity has been criticized (Flores, Gee, and Kastner 2000).

An increase in training efforts can be expected. In 1999, the American Academy of Pediatrics' Committee on Pediatric Workforce issued a policy statement on the importance of cultural competency in training in medical school, residency, and continuing medical education. Bills have recently been introduced into the New Jersey and New York legislatures that require cultural competency training as a condition of physician licensure (New Jersey State Senate 2000; New York State Assembly 1999). The Liaison Committee on Medical Education (LCME), which accredits medical schools, has proposed standards on cultural diversity, stating that medical students should understand diverse cultural health belief systems; comprehend differing epidemiology, symptomatology, and treatment responses; and learn to recognize and address cultural biases in health care delivery (LCME 2000).

However, not all training is equally effective. Studies have shown that widely used continuing medical education methods, especially shorter courses and didactic approaches, are ineffective at changing trainees' behavior (Davis et al. 1995; Davis et al. 1999; Levinson and Roter 1993). Training can also be uneven. One study of medical school cultural competency training showed improvements in knowledge in one area but not in another (Copeman 1989). The scant research that has been conducted on cultural competency training has yet to identify the components that are effective in promoting culturally competent health care and how to sustain benefits over time (Loudon et al. 1999). Research is also needed to determine which techniques have the greatest impact on minority patients' health status, functioning, and outcomes and not just trainees' satisfaction, knowledge, and attitudes.

\section{COORDINATING WITH TRADITIONAL HEALERS}

Traditional healers may function as a substitute for a small proportion of patients, but in the vast majority of cases, traditional healers are used in conjunction with the conventional health care system (Druss and Rosenheck 1999; Fadiman 1997; Higginbotham, Trevino, and Ray 1990; Kim and Kwok 1998; Ma 1999; Skaer et al. 1996; Zuckerman et al. 1996). Many times, however, clinicians are not aware that their patients are seeing traditional healers. Surveys of various populations seeing both traditional healers and biomedical clinicians indicate that anywhere from 20 to 85 percent do not tell their clinicians about 
their patronage of traditional healers (Druss and Rosenheck 1999; Elder, Gillcrist, and Minz 1997; Keegan 1996; Marbella et al. 1998). Lack of cooperation between the two types of practicioners is feared to lead to conflicts for dual users of conventional and traditional medicine (Pang 1989). One study cited that a third of patients who both saw traditional healers and attended an Indian Health Service clinic reported receiving different advice from their physicians and healers (Marbella et al. 1998). Furthermore, the patients rated their healer's advice higher than their physician's advice 61.4 percent of the time.

Learning about the use of traditional healers sometimes presents a challenge. For example, Latino focus group participants who saw alternative practitioners reported that they feared physicians would stop treating them upon learning about the alternative practitioners (Tirado 1996). Expressing familiarity with cultural beliefs, folk illnesses, and traditional practices and open nonjudgmental questioning may be able to overcome reluctance (Elder, Gillcrist, and Minz 1997; Uzbek 1994 *PLEASE PROVIDE REFERENCE*).

Increased awareness of the use of traditional healers is the first step in coordinating with them. More advanced methods include the development of referral arrangements, direct collaboration with traditional healers, and incorporation of traditional healers into health systems (Pachter 1994). These methods are relatively rare, but isolated examples exist. An example of referral relationships is provided by a clinician in Denver who refers patients to traditional healers when fear or suspicion obstruct conventional care (Kilborn 1999). Reciprocal referrals are reported by Pachter (1994), who after sending for a santiguadora (Puerto Rican folk healer) to treat a hospitalized child, was called in to treat another sick child in the community. However, no evidence on the impact of the development of referral arrangements could be located.

Research on the effects of direct collaboration with traditional healers or incorporating them into health systems' networks was also not found. While other countries have recognized traditional healers as legitimate health care providers - for example, Zimbabwe licenses traditional healers (Barrett 1996) recognition and coverage of nonconventional therapies in the United States has been slower and uneven. A 1999 survey showed that 31 percent of all health maintenance organizations cover acupuncture, but only 4 percent cover herbal therapies or homeopathy (National Market Measures 1999).

\section{COMMUNITY HEALTH WORKERS}

Community health worker programs in the United States have been adapted from the international arena. Community health workers are com- 
monly used in developing countries to extend the reach of limited health systems. The many names by which domestic community health workers goneighborhood worker, indigenous health worker, lay health adviser, consejera, promotora-are indicative of varying conceptualizations of who community health workers are and what they do.

A common definition of community health workers is that they are members of the community who work outside established health care locales to serve as connectors between populations that have been traditionally underserved and health care providers (Witmer et al. 1995). However, in practice, community health worker programs are varied. At one end of the spectrum, some have employed community health workers who come from similar communities but do not necessarily reside in the area in which they work (Krieger et al. 1999) or operate in a clinic rather than community setting (Margolis et al. 1998). In contrast, the lay health adviser model uses people in the community to whom others naturally turn for advice and help, strengthening existing community networks (D. W. Baker et al. 1997; Jackson and Parks 1997). Activities of community health workers include holding group meetings, home visitation, facilitating appointment keeping, distributing health promotion information, conducting screenings, and identifying community problems and developing solutions (Earp and Flax 1999; Krieger et al. 1999; Witmer et al. 1995). Training for community health workers varies widely, from less than 20 hours to more than 100 hours (Jackson and Parks 1997).

Despite the fact that community health worker programs have been in wide operation since the 1960s, Giblin's 1989 review of program evaluations found only one in which tests of statistical significance were used. Fortunately, in the past several years, a number of studies using control group comparisons have supplied evidence that community health workers can improve, although sometimes modestly, access to and utilization of health care. These improvements include increases in breast screening practices (Bird et al. 1998; Margolis et al. 1998; Navarro et al. 1998; Slater et al. 1998; Sung et al. 1997), higher retention in patient education programs (Corkery et al. 1997), increased follow-up (Krieger et al. 1999), and better cervical cancer knowledge and / or screening practices (Bird et al. 1998; Dignan et al. 1998; Margolis et al. 1998; Navarro et al. 1998).

The use of community health workers is not a simple undertaking. Uneven program impact and frustrations experienced by the community health workers indicate that such programs must be designed carefully (Messer, Steckler, and Dignan 1999; Rodney et al. 1998; Sung et al. 1997). However, the literature provides only the most general guidance to program developers as to what program features and community conditions are critical. 


\section{CULTURALLY COMPETENT HEALTH PROMOTION}

Cultural competency can also increase the value of health promotion activities directed at racial and ethnic minority groups. Health promotion activities, including brief clinical interventions, can be at least modestly successful in reducing harmful behaviors (e.g., smoking, excessive consumption of alcohol, poor diet) and increasing screenings (Dowswell et al. 1996; Eaton and Menard 1998; Farquhar et al. 1990; Fiore, Fleming, and Burns 1999; Kok, van den Borne, and Mullen 1997; Marcus and Crane 1998; Ritvo et al. 1997; Tang et al. 1998).

Health promotion approaches designed for majority populations may not always work as well for minority Americans (Flaskerud and Uman 1993; Guidry and Walker 1999; Mohrmann et al. 2000; Nickens 1990; Oomen, Owen, and Suggs 1999; Roetzheim et al. 1993). For example, Fletcher and colleagues (1993) discuss a community-wide intervention aimed at increasing mammography screening rates that was less successful for black women than for white women. Several factors can account for a differential impact. First, implementation may not be uniform across all populations. Studies indicate that mammography is not being recommended as frequently to black and Hispanic women as to white women (Caplan, Wells, and Haynes 1992). African Americans and Hispanics are less likely to be told by their doctors to stop smoking, and African Americans are less likely to use formal cessation programs (Hymowitz et al. 1996). Second, written health promotion materials are often deficient for minority populations. Reviews of printed cancer education materials found a majority of them to be culturally insensitive or otherwise inappropriate for minority populations (Guidry and Walker 1999; Mohrmann et al. 2000).

Third, diverse racial and ethnic groups may process these messages differently because their particular culture affects health beliefs and behaviors (Pérez-Stable, Marin, and Posner 1998). For example, differences among races in breast cancer knowledge, beliefs, and attitudes affect cancer screening and prevention behaviors and have been linked to minority women's late-stage presentation of cancer and consequent higher mortality (Lannin et al. 1998). Dietary practices, which affect cancer risk, vary among racial and ethnic groups (Otero-Sabogal et al. 1995). Culturally competent approaches have therefore been designed to take into account cultural beliefs and values to increase the likelihood that health promotion messages will be heard and heeded.

While theory and logic would suggest that cultural competency can improve the impact of health promotion efforts, studies to date have not substantiated this relationship. Many studies show that culturally competent health promotion programs increase awareness and knowledge of health prevention and promotion opportunities (e.g., smoking cessation, breast and 
cervical cancer screenings, heart disease prevention, diabetes education), but such research either did not measure behavior changes or found that the programs did not produce consistent improvements (Alcalay et al. 1999; Griffin et al. 1999; Jenkins et al. 1999; Marín and Pérez-Stable 1995). One study demonstrated that a culturally competent behavioral smoking cessation program for Hispanics was more successful at the 6-month follow-up than a self-help program, but it found that these differences eroded by the 12-month follow-up (Nevid and Javier 1997).

Even research that has demonstrated that culturally competent health promotion interventions can be effective with minority populations has not been able to conclude that their efficacy is due to the emphasis on cultural competency. Several studies have shown that when compared to no intervention, culturally competent interventions with minority women can raise breast cancer screening rates (Erwin et al. 1999; Fernandez et al. 1999; McAlister et al. 1995; Weber and Reilly 1997). A study using pre-post comparisons of a breast-feeding promotion program that reinforced traditional understandings about infant feeding documented increased breast-feeding among Navajo women (Wright et al. 1997). However, none of these studies compared culturally competent techniques to similar interventions that are not culturally competent. Targeting interventions to racial and ethnic minorities could be expected to reduce disparities, but the literature has not proved conclusively that these interventions must be culturally competent to be successful (Arblaster et al. 1996). For example, immunization rates for minority children have been increased through interventions that were not particularly culturally competent (Hoekstra et al. 1998; Wood et al. 1998). An even more dramatic example is provided by the reduction of disparities through a nonculturally competent breast cancer screening invention. In the randomized trial, the control group's survival rates mirrored racial and ethnic disparities documented in the literature, while there was no differential in the survival rates of nonwhite and white women in the intervention group (Shapiro et al. 1982).

\section{CONCLUSION}

The pervasive presence of major racial and ethnic disparities in health presents a serious public policy problem requiring multiple approaches. While improvements in economic and social conditions and the physical environment would in all likelihood make substantial contributions to reducing health disparities, health services interventions can also reduce inequalities (Arblaster et al. 1996; Schur, Albers, and Berk 1995). Our review of the literature on disparities and cultural competency provides strong reason to believe that careful and appropriate implementation of sound cultural competency 


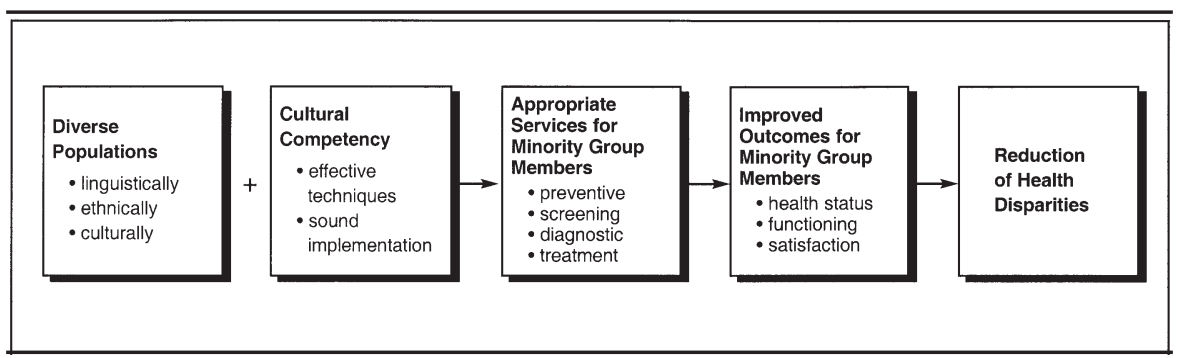

FIGURE 3 Reducing Health Disparities Through the Implementation of Cultural Competency

techniques in delivering health services could go a long way toward reducing disparities, as shown in Figure 3.

Unfortunately, at this point there is little by way of rigorous research evaluating the impact of particular cultural competency techniques on any outcomes, including the reduction of racial and ethnic disparities. The only exception is that subset of techniques related to overcoming language barriers. Most linkages among cultural competency techniques, the processes of health care service delivery, and patient outcomes have yet to be empirically tested. Comparisons of culturally competent interventions with interventions uninformed by patients' language and culture are particularly critical, given that the research literature has not been able to firmly rule out the confounders of education, literacy, and class as causes for racial and ethnic disparities.

Amassing this evidence is an essential step if cultural competency is to be widely adopted by health systems. Health systems are struggling to improve their services to minority populations. They are being held more accountable for their patient outcomes and therefore want proof that cultural competency "works" (Coye and Alvarez 1999; Lavizzo-Mourey and Mackenzie 1996). Although there is substantial research to suggest that cultural competency should work, health systems have little evidence about which cultural competency techniques are in fact effective and less evidence on how to implement them properly. Like anything else, cultural competency techniques can be sound or unsound, done well or not well. Rigorous research on cultural competency would both enable the testing of cultural competency's theoretical premises and provide health systems with constructive information about which techniques are most successful and under what circumstances.

\section{NOTES}

1. The term disease as used in this article includes physical or mental impairments that are the result of injury and violence as well as those that are biologic in their genesis. 
2. Studies designed to establish such a connection need to be conducted using objective measures, since perceived improvements in communication may not translate into actual improvements. D. W. Baker et al. (1996) found that although Latinos with a language match were more likely to believe that they understand their disease and recommended treatment better than those who used interpreters and those who thought interpreters were needed, objective measures of understanding diagnosis and treatment revealed that all groups had poor knowledge and differences among groups were not statistically significant.

\section{REFERENCES}

Adams, D.L., ed. 1995. Health Issues for Women of Color: A Cultural Diversity Perspective. Thousand Oaks, CA: Sage.

Agency for Health Care Policy and Research. 1999. Understanding and Eliminating Minority Health Disparities (RFA: HS-00-003). Rockville, MD: Agency for Health Care Policy and Research.

Alcalay, R., M. Alvarado, H. Balcazar, E. Newman, and E. Huerta. 1999. Salud para su Corazon: ACommunity-Based Latino Cardiovascular Disease Prevention and Outreach Model. Journal of Community Health 24 (5): 359-79.

American Academy of Pediatrics Committee on Pediatric Workforce. 1999. Culturally Effective Pediatric Care: Education and Training Issues. Pediatrics 103 (1): 167-70.

Andrulis, D. P., T. L. Delbanco, and Y. Shaw-Taylor. 1999. Cross Cultural Competence in Health Care Survey. Washington, DC: National Public Health and Hospital Institute.

Arblaster, L., M. Lambert, V. Entwistle, M. Forster, D. Fullerton, T. Sheldon, and I. Watt. 1996. A systematic review of the effectiveness of health service interventions aimed at reducing inequalities in health. Journal of Health Services Research E Policy 1 (2): 93-103.

Ashing-Giwa, K. 1999. Health Behavior Change Models and Their Socio-Cultural Relevance for Breast Cancer Screening in African American Women. Women and Health 28 (4): 53-71.

Association of Asian Pacific Community Health Organizations. 1996. State Medicaid Managed Care: Requirements for Linguistically Appropriate Health Care. Oakland, CA: Association of Asian Pacific Community Health Organizations.

Association of State and Territorial Health Officials. 1994. Multicultural Public Health Capacity Building Pilot Projects: Final Report. U.S. Department of Health and Human Services, Office of Minority Health.

Ayanian, J. Z., S. Udvarhelyi, C. A. Gatsonis, C. L. Pashos, and A. M. Epstein. 1993. Racial Differences in the Use of Revascularization Procedures After Coronary Angiography. Journal of the American Medical Association 269 (20): 2642-46.

Baker, D. W., R. M. Parker, M. V. Williams, W. C. Coates, and K. Pitkin. 1996. Use and Effectiveness of Interpreters in an Emergency Department. Journal of the American Medical Association 275 (10): 783-8. 
Baker, D. W., R. Hayes, and J. P. Fortier. 1998. Interpreter Use and Satisfaction with Interpersonal Aspects of Care for Spanish-Speaking Patients. Medical Care 36 (10): 1461-70.

Baker, E. A., N. Bouldin, M. Durham, M. E. Lowell, M. Gonzalez, N. Jodaitis, L. N. Cruz, I. Torres, M. Torres, and S. T. Adams. 1997. The Latino Health Advocacy Program: A Collaborative Lay Health Advisor Approach. Health Education E Behavior 24 (4): 495-509.

Baquet, C. R., and P. Commiskey. 1999. Colorectal Cancer Epidemiology in Minorities: A Review. Journal of the Association of Academic Minority Physicians 10 (3): 51-8.

Barrett, S. 1996. Zimbabwe Uses All Medical Resources to Find Solutions. AIDS Analysis Africa 6 (1): 13.

Bird, J. A., S. J. McPhee, N. T. Ha, B. Le, T. Davis, and C. N. Jenkins. 1998. Opening Pathways to Cancer Screening for Vietnamese-American Women: Lay Health Workers Hold a Key. Preventive Medicine 27 (6): 821-29.

Blackhall, L. J., S. T. Murphy, G. Frank, V. Michel, and S. Azen. 1995. Ethnicity and attitudes toward patient autonomy. Journal of the American Medical Association 274 (10): 820-5.

Cameron, C. 1996. Patient compliance: recognition of factors involved and suggestions for promoting compliance with therapeutic regimens. Journal of Advanced Nursing 24: 244-50.

-1999. How Does Cultural Competence Affect Patient Safety? Focus on Patient Safety 2 (4): 3, 6.

Caplan, L. S., B. L. Wells, and S. Haynes. 1992. Breast Cancer Screening among Older Racial/Ethnic Minorities and Whites: Barriers to Early Detection. Journal of Gerontology 47: 101-10.

Carlisle, D. M., B. D. Leake, and M. F. Shapiro. 1997. Racial and Ethnic Disparities in the Use of Cardiovascular Procedures: Associations with Type of Health Insurance. American Journal of Public Health 87 (2): 263-7.

Carrasquillo, O., E. J. Orav, T. A. Brennan, and H. R. Burstin. 1999. Impact of Language Barriers on Patient Satisfaction in an Emergency Department. Journal of General Internal Medicine 14 (2): 82-7.

Chang, P. H., and J. P. Fortier. 1998. Language Barriers to Health Care: An Overview. Journal of Health Care for the Poor and Underserved 9 (Supplement): S5-19.

Collins, K. S., A. Hall, and C. Neuhaus. 1999. U.S. Minority Health: A Chartbook. New York: Commonwealth Fund.

Commonwealth Fund. 1995. National Comparative Survey of Minority Health Care. New York: Commonwealth Fund.

Conigliario, J., J. Whittle, C. B. Good, B. H. Hanusa, L. J. Passman, R. P. Lofgren, R. Allman, P. A. Ubel, M. O'Connor, and D. S. Macpherson. 2000. Understanding Racial Variation in the Use of Coronary Revascularization Procedures: The Role of Clinical Factors. Archives of Internal Medicine 160 (9): 1329-35.

Cooper-Patrick, L., J. J. Gallo, J. J. Gonzales, H. T. Vu, N. R. Powe, C. Nelson, and D. E. Ford. 1999. Race, Gender, and Partnership in the Patient-Physician Relationship. Journal of the American Medical Association 282 (6): 583-9. 
Copeman, R. C. 1989. Medical Students, Aborigines and Migrants: Evaluation of a Teaching Programme. Medical Journal of Australia 150 (2): 84-7.

Corkery, E., C. Palmer, M. E. Foley, C. B. Schechter, L. Frisher, and S. H. Roman. 1997. Effect of a Bicultural Community Health Worker on Completion of Diabetes Education in a Hispanic Population. Diabetes Care 20 (3): 254-57.

Coye, M., and D. Alvarez. 1999. Medicaid Managed Care and Cultural Diversity in California. New York: Commonwealth Fund.

Cross, T. L., B. J. Bazron, K. W. Dennis, and M. R. Isaacs. 1989. Towards a Culturally Competent System of Care: A Monograph on Effective Services for Minority Children Who Are Severely Emotionally Disturbed. Washington, DC: CASSP Technical Assistance Center, Georgetown University Child Development Center.

Culhane-Pera, K. A., C. Reif, E. Egli, N. J. Baker, and R. Kassekert. 1997. A Curriculum for Multicultural Education in Family Medicine. Family Medicine 29 (10): 719-23.

Culhane-Pera, K. A., R. C. Like, P. Lebensohn-Chialvo, and R. Loewe. 2000. Multicultural Curricula in Family Practice Residencies. Family Medicine 32 (3): 167-73.

D' Andrea, M., J. Daniels, and R. Heck. 1991. Evaluating the Impact of Multicultural Counseling Training. Journal of Counseling and Development 70 (September/October): $143-50$.

D' Avanzo, C. E. 1992. Barriers to Health Care for Vietnamese Refugees. Journal of Professional Nursing 8 (4): 245-53.

David, R. A., and M. Rhee. 1998. The Impact of Language as a Barrier to Effective Health Care in an Underserved Urban Hispanic Community. Mount Sinai Journal of Medicine 65 (5, 6): 393-7.

Davis, D. A., M. A. Thomson, A. D. Oxman, and R. B. Haynes. 1995. Changing Physician Performance: A Systematic Review of the Effect of Continuing Medical Education Strategies. Journal of the American Medical Association 274 (9): 700-5.

Davis, D., M.A.T. O’ Brien, N. Freemantle, F. M. Wolf, P. Mazmanian, and A. Taylor-Vaisey. 1999. Impact of Formal Continuing Medical Education: Do Conferences, Workshops, Rounds, and Other Traditional Continuing Education Activities Change Physician Behavior or Health Care Outcomes? Journal of the American Medical Association 282 (9): 867-74.

Denboba, D. L., J. L. Bragdon, L. G. Epstein, K. Garthright, and T. M. Goldman. 1998. Reducing Health Disparities through Cultural Competence. Journal of Health Education 29 (5, suppl.): S47-53.

Derose, K. P., and D. W. Baker. 2000. Limited English Proficiency and Latinos' Use of Physician Services. Medical Care Research and Review 57 (1): 76-91.

Dignan, M. B., R. Michielutte, H. B. Wells, P. Sharp, K. Blinson, L. D. Case, R. Bell, J. Konen, S. Davis, and R. P. McQuellon. 1998. Health Education to Increase Screening for Cervical Cancer among Lumbee Indian women in North Carolina. Health Education Research 13 (4): 545-56.

Dowswell, T., E. M. Towner, G. Simpson, and S. N. Jarvis. 1996. Preventing Childhood Unintentional Injuries-What Works? A Literature Review. Injury Prevention 2 (2): 140-9. 
Druss, B. G., and R. A. Rosenheck. 1999. Association Between Use of Unconventional Therapies and Conventional Medical Services. Journal of the American Medical Association 282 (7): 651-6.

Earp, J. A., and V. L. Flax. 1999. What Lay Health Advisors Do: An Evaluation of Advisors' Activities. Cancer Practice 7 (1): 16-21.

East, M. A., and E. D. Peterson. 2000. Understanding Racial Differences in Cardiovascular Care and Outcomes: Issues for the New Millenium. American Heart Journal 139:764-6.

Eaton, C. B., and L. M. Menard. 1998. A Systematic Review of Physical Activity Promotion in Primary Care Office Settings. British Journal of Sports Medicine 32 (1): 11-6.

Egbert, L. D., and I. L. Rothman. 1977. Relation between the Race and Economic Status of Patients and Who Performs Their Surgery. New England Journal of Medicine 297 (2): 90-1.

Elder, N. C., A. Gillcrist, and R. Minz. 1997. Use of Alternative Health Care by Family Practice Patients. Archives of Family Medicine 6 (2): 181-4.

Enguidanos, E. R., and P. Rosen. 1997. Language as a Factor Affecting Follow-up Compliance from the Emergency Department. Journal of Emergency Medicine 15 (1): 9-12.

Eraker, S. A., J. P. Kirscht, and M. H. Becker. 1984. Understanding and Improving Patient Compliance. Annals of Internal Medicine 100 (2): 258-68.

Erwin, D. O., T. S. Spatz, R. C. Stotts, and J. A. Hollenberg. 1999. Increasing Mammography Practice by African American Women. Cancer Practice 7 (2): 78-85.

Erzinger, S. 1991. Communication between Spanish-Speaking Patients and Their Doctors in Medical Encounters. Culture, Medicine, and Psychiatry 15:91-110.

Facione, N. C. 1999. Breast Cancer Screening in Relation to Access to Health Services. Oncology Nursing Forum 26 (4): 689-96.

Fadiman, A. 1997. The Spirit Catches You and You Fall Down: A Hmong Child, Her American Doctors, and the Collision of Two Cultures. New York: Farrar, Straus \& Giroux.

Farquhar, J. W., S. P. Fortmann, J. A. Flora, C. B. Taylor, W. L. Haskell, P. T. Williams, N. Maccoby, and P. D. Wood. 1990. Effects of Communitywide Education on Cardiovascular Disease Risk Factors: The Stanford Five-City Project. Journal of the American Medical Association 264 (3): 359-65.

Ferguson, J. A., M. Weinberger, G. R. Westmoreland, L. A. Mamlin, D. S. Segar, J. Y. Greene, D. K. Martin, and W. M. Tierney. 1998. Racial Disparity in Cardiac Decision Making. Archives of Internal Medicine 158 (13): 1450-3.

Fernandez, M. E., M. DeBor, M. J. Candreia, K. Wagner, and K. R. Stewart. 1999. Evaluation of ENCOREplus. A Community-Based Breast and Cervical Cancer Screening Program. American Journal of Preventive Medicine 16 (3S): 35-49.

Fiore, M. C., M. F. Fleming, and M. E. Burns. 1999. Tobacco and Alcohol Abuse: Clinical Opportunities for Effective Intervention. Proceedings of the Association of American Physicians 111 (2): 131-40.

Flaskerud, J. H., and P. Y. Liu. 1991. Effects of an Asian Client-Therapist Language, Ethnicity and Gender Match on Utilization and Outcome of Therapy. Community Mental Health Journal 27 (1): 31-42. 
Flaskerud, J. H., and G. Uman. 1993. Directions for AIDS Education for Hispanic Women Based on Analyses of Survey Findings. Public Health Reports 108 (3): 298-304.

Fletcher, S. W., R. P. Harris, J. J. Gonzalez, D. Degnan, D. R. Lannin, V. J. Strecher, C. Pilgrim, D. Quade, J. A. Earp, and R. L. Clark. 1993. Increasing Mammography Utilization: A Controlled Study. Journal of the National Cancer Institute 85 (2): 112-20.

Flores, G., M. Abreu, M. A. Olivar, and B. Kastner. 1998. Access Barriers to Health Care for Latino Children. Archives of Pediatrics and Adolescent Medicine 152 (11): 1119-25.

Flores, G., D. Gee, and B. Kastner. 2000. The Teaching of Cultural Issues In U.S. and Canadian Medical Schools. Academic Medicine 75 (5): 451-5.

Flores, G., and L. R. Vega. 1998. Barriers to Health Care Access for Latino Children: A Review. Family Medicine 30 (3): 196-205.

Ford, E. S., and R. S. Cooper. 1995. Implications of Race/Ethnicity for Health and Health Care Use. Health Services Research 30 (1): 237-52.

Fortier, J. P., and Y. Shaw-Taylor. 1999. Cultural and Linguistic Competence Standards And Research Agenda Project: Part One. Recommendations for National Standards. Silver Spring, MD: Resources for Cross Cultural Health Care.

Frederick Schneiders Research. 1999. Perceptions of How Race and Ethnic Background Affect Medical Care: Highlights from Focus Groups. Menlo Park, CA: The Henry J. Kaiser Family Foundation.

Gaston, R. S., I. Ayres, L. G. Dooley, and A. G. Diethelm. 1993. Racial Equity in Renal Transplantation. The Disparate Impact of Hla-Based Allocation. Journal of the American Medical Association 270 (11): 1352-6.

Giblin, P. T. 1989. Effective Utilization and Evaluation of Indigenous Health Care Workers. Public Health Reports 104 (4): 361-8.

Goicoechea-Balbona, A. 1997. Culturally Specific Health Care Model for Ensuring Health Care Use by Rural, Ethnically Diverse Families Affected by HIV/AIDS. Health and Social Work 22 (3): 172-80.

Goldberg, K. C., A. J. Hartz, S. J. Jacobsen, H. Krakauer, and A. A. Rimm. 1992. Racial and Community Factors Influencing Coronary Artery Bypass Graft Surgery Rates for All 1986 Medicare Patients. Journal of the American Medical Association 267 (11): 1473-7.

Gordon, A. K. 1995. Deterrents to Access and Service for Blacks and Hispanics: The Medicare Hospice Benefit, Healthcare Utilization, and Cultural Barriers. The Hospice Journal 10 (2): 65-83.

Gornick, M. E., P. W. Eggers, T. W. Reilly, R. M. Mentnech, L. K. Fitterman, L. E. Kucken, and B. C. Vladeck. 1996. Effects of Race and Income on Mortality and Use of Services among Medicare Beneficiaries. New England Journal of Medicine 335 (11): 791-9.

Grantmakers in Health. 1998. Chartbook: Eliminating Racial and Ethnic Disparities in Health. Paper read at Grantmakers in Health, 11 September 1998, Potomac, MD.

Griffin, J. A., S. S. Gilliland, G. Perez, D. Helitzer, and J. S. Carter. 1999. Participant Satisfaction with a Culturally Appropriate Diabetes Education Program: The Native American Diabetes Project. Diabetes Educator 25 (3): 351-63. 
Gropper, R. C. 1998. Cultural Basics and Chronic Illness. Advances in Renal Replacement Therapy 5 (2): 128-33.

Guidry, J. J., and V. D. Walker. 1999. Assessing Cultural Sensitivity in Printed Cancer Materials. Cancer Practice 7 (6): 291-6.

Hampers, L. C., S. Cha, D. J. Gutglass, H. J. Binns, and S. E. Krug. 1999. Language Barriers and Resource Utilization in a Pediatric Emergency Department. Pediatrics 103 (6): 1253-6.

Heurtin-Roberts, S., and E. Reisin. 1992. The Relation of Culturally Influenced Lay Models of Hypertension to Compliance with Treatment. American Journal of Hypertension 5 (11): 787-92.

Higginbotham, J. C., F. M. Trevino, and L. A. Ray. 1990. Utilization of Curanderos by Mexican-Americans: Prevalence and Predictors: Findings from HHANES 1982-84. American Journal of Public Health 80 (suppl.): 32-5.

Hoekstra, E. J., C. W. LeBaron, Y. Megaloeconomou, H. Guerrero, C. Byers, T. Johnson-Partlow, B. Lyons, E. Mihalek, J. Devier, and J. Mize. 1998. Impact of a Large-Scale Immunization Initiative in the Special Supplemental Nutrition Program for Women, Infants and Children (WIC). Journal of the American Medical Association 280 (13): 1143-7.

Hornberger, J. C., C. D. Gibson, W. Wood, C. Dequeldre, I. Corso, B. Palla, and D. A. Bloch. 1996. Eliminating Language Barriers for Non-English-Speaking Patients. Medical Care 34 (8): 845-56.

Howard, D. L., R. Penchansky, and M. B. Brown. 1998. Disaggregating the Effects of Race on Breast Cancer Survival. Family Medicine 30 (3): 228-35.

Hurley, J. S., F. J. Frost, K. M. Trinkaus, M. C. Buatti, and K. E. Emmett. 1998. Relationship of Compliance with Hormone Replacement Therapy to Short-Term Healthcare Utilization in a Managed Care Population. American Journal of Managed Care 4 (12): 1691-8.

Hymowitz, N., J. Jackson, R. Carter, and H. Eckholdt. 1996. Past Quit Smoking Assistance And Doctors' Advice for White and African-American Smokers. Journal of the American National Medical Association 88 (4): 249-52.

Jackson, E. J., and C. P. Parks. 1997. Recruitment and Training Issues from Selected Lay Health Advisor Programs among African Americans: A20-year Perspective. Health Education \& Behavior 24 (4): 418-31.

Jenkins, C. N., S. J. McPhee, J. A. Bird, G. Q. Pham, B. H. Nguyen, T. Nguyen, K. Q. Lai, C. Wong, and T. B. Davis. 1999. Effect of a Media-led Education Campaign on Breast and Cervical Cancer Screening among Vietnamese-American Women. Preventive Medicine 28 (4): 395-406.

Joos, S. K., D. H. Hickam, G. H. Gordon, and L. H. Baker. 1996. Effects of a Physician Communication Intervention on Patient Care Outcomes. Journal of General Internal Medicine 11 (3): 147-55.

Kaiser Family Foundation. 1999. Race, Ethnicity and Medical Care: A Survey of Public Perceptions and Experiences. Menlo Park, CA: The Henry J. Kaiser Family Foundation.

Kaplan, S. H., S. Greenfield, and J. Ware. 1989. Assessing the Effects of Physician-Patient Interactions on the Outcomes of Chronic Disease. Medical Care 27 (3): S110-27. 
Kavanagh, K., K. Absalom, J. W. Beil, and L. Schliessmann. 1999. Connecting and Becoming Culturally Competent: A Lakota Example. Advances in Nursing Science 21 (3): 9-31.

Keegan, L. 1996. Use of Alternative Therapies among Mexican Americans in the Texas Rio Grande Valley. Journal of Holistic Nursing 14 (4): 277-94.

Kennedy, S., B. Stubblefield-Tave, and C. Smith. 1999. Report on Recommendations for Measures of Cultural Competence for the Quality Improvement System for Managed Care. Cambridge, MA: Abt Associates.

Kilborn, P. T. 1999. Denver's Hispanic residents point to ills of the uninsured. New York Times, 9 April 9, p. A1.

Kim, C., and Y. S. Kwok. 1998. Navajo Use of Native Healers. Archives of Internal Medicine 158 (20): 2245-9.

Kington, R. S., and J. P. Smith. 1997. Socioeconomic Status and Racial and Ethnic Differences in Functional Status Associated with Chronic Diseases. American Journal of Public Health 87 (5): 805-10.

Kleinman, A., L. Eisenberg, and B. Good. 1978. Culture, Illness, and Care: Clinical Lessons from Anthropologic and Cross-Cultural Research. Annals of Internal Medicine 88 (2): 251-8.

Kok, G., B. van den Borne, and P. D. Mullen. 1997. Effectiveness of Health Education and Health Promotion: Meta-Analyses of Effect Studies and Determinants of Effectiveness. Patient Education and Counseling 30 (1): 19-27.

Komaromy, M., K. Grumbach, M. Drake, K. Vranizan, N. Lurie, D. Keane, and A. B. Bindmand. 1996. The Role of Black and Hispanic Physicians in Providing Health Care for Underserved Populations. New England Journal of Medicine 334 (20): 1305-10.

Krieger, J., C. Collier, L. Song, and D. Martin. 1999. Linking Community-based Blood Pressure Measurement to Clinical Care: A Randomized Controlled Trial of Outreach and Tracking by Community Health Workers. American Journal of Public Health 89 (6): 856-61.

Lane, J. 1998. An Enduring Partnership: Kaiser Permanente and Su Salud. The Permanente Journal 2 (3). Available: http://www.kaiserpermanente.org/medicine/permjournal/permjournal.html

Lannin, D. R., H. F. Mathews, J. Mitchell, M. S. Swanson, F. H. Swanson, and M. S. Edwards. 1998. Influence of Socioeconomic and Cultural Factors on Racial Differences in Late-Stage Presentation of Breast Cancer. Journal of the American Medical Association 279 (22): 1801-7.

Lavizzo-Mourey, R., and E. R. Mackenzie. 1996. Cultural Competence: Essential Measurements of Quality for Managed Care Organizations. Annals of Internal Medicine 124 (10): 919-20.

Lawson, W. B. 1996. The Art and Acience of the Psychopharmacotherapy of African Americans. Mount Sinai Journal of Medicine 63 (5-6): 301-5.

Levinson, W., and D. Roter. 1993. The Effects of Two Continuing Medical Education Programs on Communication Skills of Practicing Primary Care Physicians. Journal of General Internal Medicine 8 (6): 318-24. 
Liaison Committee on Medical Education. 2000. LCME accreditation standards. Liaison Committee on Medical Education.

Libby, D. L., Z. Zhou, and D. A. Kindig. 1997. Will Minority Physician Supply Meet U.S. Needs? Projections for Reaching Racial Parity of Physicians to Population. Health Affairs 16 (4): 205-14.

Lillie-Blanton, M., and T. Laveist. 1996. Race/Ethnicity, the Social Environment, and Health. Social Science and Medicine 43 (1): 83-91.

Lipton, R. B., L. M. Losey, A. Giachello, J. Mendez, and M. H. Girotti. 1998. Attitudes and Issues in Treating Latino Patients with Type 2 Diabetes: Views of Healthcare Providers. The Diabetes Educator 24 (1): 67-71.

Loudon, R. F., P. M. Anderson, P. S. Gill, and S. M. Greenfield. 1999. Educating Medical Students for Work in Culturally Diverse Societies. Journal of the American Medical Association 282 (9): $875-80$.

Ma, G. X. 1999. Between Two Worlds: The Use of Traditional and Western Health Services by Chinese immigrants. Journal of Community Health 24 (6): 421-37.

Manson, A. 1988. Language Concordance as a Determinant of Patient Compliance and Emergency Room Use in Patients with Asthma. Medical Care 26 (12): 1119-28.

Marbella, A. M., M. C. Harris, S. Diehr, G. Ignace, and G. Ignace. 1998. Use of Native American Healers among Native American Patients in an Urban Native American Health Center. Archives of Family Medicine 7 (2): 182-5.

Marcos, L. R. 1979. Effects of Interpreters on the Evaluation of Psychopathology in Non-English Speaking Patients. American Journal of Psychiatry 136 (2): 171-4.

Marcus, A. C., and L. A. Crane. 1998. A Review of Cervical Cancer Screening Intervention Research: Implications for Public Health Programs and Future Research. Preventive Medicine 27 (1): 13-31.

Margolis, K. L., N. Lurie, P. G. McGovern, M. Tyrrell, and J. S. Slater. 1998. Increasing Breast and Cervical Cancer Screening in Low-Income Women. Journal of General Internal Medicine 13 (8): 515-21.

Marín, G., and E. J. Pérez-Stable. 1995. Effectiveness of Disseminating Culturally Appropriate Smoking-Cessation Information: Programa Latino Para Dejar de Fumar. Journal of the National Cancer Institute Monographs 18:155-63.

Marvel, M. K., M. Grow, and P. Morphew. 1993. Integrating Family and Culture into Medicine: A Family Systems Block Rotation. Family Medicine 25 (7): 441-2.

Mayberry, R. M., F. Mili, I.G.M. Vaid, A. Samadi, E. Ofili, M. S. McNeal, P. A. Griffith, and G. LaBrie. 1999. Racial and Ethnic Differences in Access to Medical Care: A Synthesis of the Literature. Menlo Park, CA: The Henry J. Kaiser Family Foundation.

Maynard, C., L. D. Fisher, E. R. Passamani, and T. Pullum. 1986. Blacks in the Coronary Artery Surgery Study (CASS): Race and Clinical Decision Making. American Journal of Public Health 76 (12): 1446-8.

McAlister, A. L., M. E. Fernandez-Esquer, A. G. Ramirez, F. Trevino, K. J. Gallion, R. Villarreal, L. V. Pulley, S. Hu, I. Torres, and Q. Zhang. 1995. Community Level Cancer Control in a Texas Barrio: Part II. Base-line and Preliminary Outcome Findings. Journal of the National Cancer Institute Monographs 18:123-6. 
Messer, L., A. Steckler, and M. Dignan. 1999. Early Detection of Cervical Cancer among Native American Women: A Qualitative Supplement to a Quantitative Study. Health Education and Behavior 26 (4): 547-62.

Moffic, H. S., and J. D. Kinzie. 1996. The History and Future of Cross-Cultural Psychiatric Services. Community Mental Health Journal 32 (6): 581-92.

Mohrmann, C. C., E. A. Coleman, S. K. Coon, J. E. Lord, J. K. Heard, M. J. Cantrell, and E. C. Burks. 2000. An Analysis of Printed Breast Cancer Information for African American Women. Journal of Cancer Education 15 (1): 23-7.

Morales, L. S., W. E. Cunningham, J. A. Brown, H. Liu, and R. D. Hays. 1999. Are Latinos Less Satisfied with Communication by Health Care Providers? Journal of General Internal Medicine 14 (7): 409-17.

Morrow-Howell, N., L. A. Chadiha, E. K. Proctor, M. Hourd-Bryant, and P. Dore. 1996. Racial Differences in Discharge Planning. Health Social Work 21 (2): 131-9.

National Market Measures Inc. 1999. The Landmark Report II on HMOs and Alternative Care. Sacramento, CA: Landmark Healthcare.

Navarro, A. M., K. L. Senn, L. J. McNicholas, R. M. Kaplan, B. Roppe, and M. C. Campo. 1998. Por La Vida Model Intervention Enhances Use of Cancer Screening Tests among Latinas. American Journal of Preventive Medicine 15 (1): 32-41.

Nevid, J. S., and R. A. Javier. 1997. Preliminary Investigation of a Culturally Specific Smoking Cessation Intervention for Hispanic Smokers. American Journal of Health Promotion 11 (3): 198-207.

New Jersey State Senate. 2000. Senate Bill 237, edited by S. W. Bryant: State of New Jersey.

New York State Assembly. 1999. Assembly Bill 11202, edited by Committee on Rules: State of New York.

Nickens, H. W. 1990. Health Promotion and Disease Prevention among Minorities. Health Affairs (Millwood) 9 (2): 133-43.

— 1992. The Rationale for Minority-Targeted Programs in Medicine in the 1990s. Journal of the American Medical Association 267 (17): 2390-5.

-1995. The Role of Race/Ethnicity and Social Class in Minority Health Status. Health Services Research 30 (1, Part II): 151-62.

Oddone, E. Z., R. D. Horner, T. Diers, J. Lipscomb, L. McIntyre, C. Cauffman, J. Whittle, L. J. Passman, L. Kroupa, R. Heaney, and D. Matchar. 1998. Understanding Racial Variation in the Use of Carotid Endarterectomy: The Role of Aversion to Surgery. Journal of the National Medical Association 90 (1): 25-33.

Oddone, E. Z., R. D. Horner, R. Sloane, L. McIntyre, A. Ward, J. Whittle, L. J. Passman, L. Kroupa, R. Heaney, S. Diem, and D. Matchar. 1999. Race, Presenting Signs and Symptoms, use of Carotid Artery Imaging, and Appropriateness of Carotid Endarterectomy. Stroke 30 (7): 1350-6.

Ong, L.M.L., J.C.J.M. De Haes, A. M. Hoos, and F. B. Lammes. 1995. Doctor-Patient Communication: A Review of the Literature. Social Science Medicine 40 (7): 903-18.

Oomen, J. S., L. J. Owen, and L. S. Suggs. 1999. Culture Counts: Why Current Treatment Models Fail Hispanic Women with Type 2 Diabetes. Diabetes Educator 25 (2): 220-5. 
Orlandi, M. A., ed. 1995. Cultural Competence for Evaluators: A Guide for Alcohol and Other Drug Abuse Prevention Practitioners Working with Ethnic/Racial Communities. 2d ed. Vol. 1. OSAP Cultural Competence Series. Rockville, MD: U.S. Department of Health and Human Services.

Otero-Sabogal, R., F. Sabogal, E. J. P!rez-Stable, and R. A. Hiatt. 1995. Dietary Practices, Alcohol Consumption, and Smoking Behavior: Ethnic, Sex, and Acculturation Differences. Journal of the National Cancer Institute Monographs 18:73-82.

Pachter, L. M. 1994. Culture and Clinical Care: Folk Illness Beliefs and Behaviors and Their Implications for Health Care Delivery. Journal of the American Medical Association 271 (9): 690-4.

Pang, K. Y. 1989. The Practice of Traditional Korean Medicine in Washington, D.C. Social Science and Medicine 28 (8): 875-84.

Peniston, R. L., D. Y. Lu, V. Papademetriou, and R. D. Fletcher. 2000. Severity of Coronary Artery Disease in Black and White Male Veterans and Likelihood of Revascularization. American Heart Journal 139 (5): 840-7.

Perkins, J., H. Simon, F. Cheng, K. Olson, and Y. Vera. 1998. Ensuring Linguistic Access in Health Care Settings: Legal Rights and Responsibilities. Los Angeles: National Health Law Program.

Perkins, J., and Y. Vera. 1998. Legal Protections to Ensure Linguistically Appropriate Health care. Journal of Health Care for the Poor and Underserved 9 (Suppl.): S62-80.

Pérez-Stable, E. J., A. Napoles-Springer, and J. M. Miramontes. 1997. The Effects of Ethnicity and Language on Medical Outcomes of Patients with Hypertension or Diabetes. Medical Care 35 (12): 1212-19.

Pérez-Stable, E. J., G. Marin, and S. F. Posner. 1998. Ethnic Comparison of Attitudes and Beliefs about Cigarette Smoking. Journal of General Internal Medicine 13 (3): 167-74.

Peterson, E. D., S. M. Wright, J. Daley, and G. E. Thibault. 1994. Racial Variation in Cardiac Procedure Use and Survival Following Acute Myocardial Infarction in the Department of Veterans Affairs. Journal of the American Medical Association 271 (15): $1175-80$

President's Advisory Commission on Consumer Protection and Quality in the Health Care Industry. 1997. Consumer Bill of Rights and Responsibilities. Washington, DC: Advisory Commission on Consumer Protection and Quality in the Health Care Industry.

Rathore, S. S., L. A. Lenert, K. P. Weinfurt, A. Tinoco, C. K. Taleghani, W. Harless, and K. A. Schulman. 2000. The Effects of Patient Sex and Race on Medical Students' Ratings of Quality of Life. American Journal of Medicine 108:561-6.

Richardson, L. D. 1999. Patients' Rights and Professional Responsibilities: The Moral Case for Cultural Competence. Mt. Sinai Journal of Medicine 66 (4): 267-70.

Riddick, S. 1998. Improving Access for Limited English-Speaking Consumers: A Review of Strategies in Health Care Settings. Journal of Health Care for the Poor and Underserved 9 (Suppl.): S40-61.

Ritvo, P. G., M. J. Irvine, E. A. Lindsay, N. Kraetschmer, N. Blair, and Z. M. Shnek. 1997. A Critical Review of Research Related to Family Physician-Assisted Smoking Cessation Interventions. Cancer Prevention and Control 1 (4): 289-303. 


\section{MCRER 57 (Supplemental: Racial and Ethnic Inequities, 2000)}

Rivadeneyra, R., V. Elderkin-Thompson, R. C. Silver, and H. Waitzkin. 2000. Patient Centeredness in Medical Encounters Requiring an Interpreter. American Journal of Medicine 108 (6): 470-4.

Robbins, A. S., A. S. Whittemore, and S. K. Van Den Eeden. 1998. Race, Prostate Cancer Survival, and Membership in a Large Health Maintenance Organization. Journal of the National Cancer Institute 90 (13): 986-90.

Rodney, M., C. Clasen, G. Goldman, R. Markert, and D. Deane. 1998. Three Evaluation Methods of a Community Health Advocate Program. Journal of Community Health 23 (5): 371-81.

Roetzheim, R. G., D. J. Van Durme, H. J. Brownlee, A. H. Herold, L. J. Woodard, and C. Blair. 1993. Barriers to Screening among Participants of a Media-Promoted Breast Cancer Screening Project. Cancer Detection and Prevention 17 (3): 367-77.

Sackett, D. L., and J. C. Snow. 1979. The Magnitude of Compliance and Noncompliance. In Compliance in Health Care, edited by R. B. Haynes, D. W. Taylor, and D. L. Sackett. Baltimore: The Johns Hopkins University Press.

Sarver, J., and D. W. Baker. 2000. Effect of Language Barriers on Follow-up Appointments after an Emergency Department Visit. Journal of General Internal Medicine 15 (4): 256-64.

Schulman, K. A., J. A. Berlin, W. Harless, J. F. Kerner, S. Sistrunk, B. J. Gersh, R. Dube, C. K. Taleghani, J. E. Burke, S. Williams, J. M. Eisenberg, and J. J. Escarce. 1999. The Effect of Race and Sex on Physicians' Recommendations for Cardiac Catheterization. The New England Journal of Medicine 340 (8): 618-26.

Schur, C. L., L. A. Albers, and M. L. Berk. 1995. Health Care Use by Hispanic Adults: Financial vs. Non-Financial Determinants. Health Care Financing Review 17 (2): 71-88.

Seijo, R., H. Gomez, and J. Freidenberg. 1991. Language as a Communication Barrier in Medical Care for Hispanic patients. Hispanic Journal of Behavioral Sciences 13 (4): 363-76.

Shapiro, J., and E. Saltzer. 1981. Cross-Cultural Aspects of Physician-Patient Communications Patterns. Urban Health 10 (10): 10-15.

Shapiro, S., W. Venet, P. Strax, L. Venet, and R. Roeser. 1982. Prospects for Eliminating Racial Differences in Breast Cancer Survival Rates. American Journal of Public Health 72 (10): 1142-5.

Sheifer, S. E., J. J. Escarce, and K. A. Schulman. 2000. Race and Sex Differences in the Management of Coronary Artery Disease. American Heart Journal 139 (5): 848-57.

Sherer, J. L. 1994. Neighbor to Neighbor: Community Health Workers Educate Their Own. Hospitals 68 (20): 52-6.

Skaer, T. L., L. M. Robison, D. A. Sclar, and G. H. Harding. 1996. Utilization of Curanderos among Foreign Born Mexican-American Women Attending Migrant Health Clinics. Journal of Cultural Diversity 3 (2): 29-34.

Slater, J. S., C. N. Ha, M. E. Malone, P. McGovern, S. D. Madigan, J. R. Finnegan, A. L. Casey-Paal, K. L. Margolis, and N. Lurie. 1998. A Randomized Community Trial to Increase Mammography Utilization among Low-Income Women Living in Public Housing. Preventive Medicine 27 (6): 862-70. 
Solis, J. M., G. Marks, M. Garcia, and D. Shelton. 1990. Acculturation, Access to Care, and Use of Preventive Services by Hispanics: Findings from HHANES 1982-1984. American Journal of Public Health 80 (Suppl.): 11-9.

St. Clair, A., and L. McKenry. 1999. Preparing Culturally Competent Practitioners. Journal of Nursing Education 38 (5): 228-34.

Stein, J. A., S. A. Fox, and P. J. Murata. 1991. The Influence of Ethnicity, Socioeconomic Status, and Psychological Barriers on Use of Mammography. Journal of Health and Social Behavior 32 (June): 101-13.

Stewart, M. A. 1995. Effective Physician-Patient Communication and Health Outcomes: A Review. Journal of the Canadian Medical Association 152 (9): 1423-33.

Stewart, M., L. Meredith, J. B. Brown, and J. Galajda. 2000. The Influence of Older Patient-Physician Communication on Health and Health-Related Outcomes. Clinical Geriatric Medicine 16 (1): 25-36.

Sung, J. F., D. S. Blumenthal, R. J. Coates, J. E. Williams, E. Alema-Mensah, and J. M. Liff. 1997. Effect of a Cancer Screening Intervention Conducted by Lay Health Workers among Inner-City Women. American Journal of Preventive Medicine 13 (1): 51-7.

Takeuchi, D. T., E. Uehara, and G. Maramba. 1999. Cultural Diversity and Mental Health Treatment. In The Sociology of Mental Health, edited by A. Horwitz and T. Scheid. New York: Oxford.

Tang, J. L., J. M. Armitage, T. Lancaster, C. A. Silagy, G. H. Fowler, and H.A.W. Neil. 1998. Systematic Review of Dietary Intervention Trials to Lower Blood Total Cholesterol in Free-Living Subjects. British Medical Journal 316 (7139): 1213-20.

Tervalon, M., and J. Murray-Garcia. 1998. Cultural Humility versus Cultural Competence: A Critical Distinction in Defining Physician Training Outcomes in Multicultural Education. Journal of Health Care for the Poor and Underserved 9 (2): 117-25.

Tirado, M. D. 1996. Tools for Monitoring Cultural Competence in Health Care. San Francisco: Latino Coalition for a Healthy California.

Tocher, T. M., and E. Larson. 1998. Quality of Diabetes Care for Non-English Speaking Patients: A Comparative Study. Western Journal of Medicine 168:504-11.

Torres, R. E. 1998. The Pervading Role of Language on Health. Journal of Health Care for the Poor and Underserved 9 (Suppl.): S21-5.

U.S. Bureau of the Census. 1996. Current Population Reports, Series P25-1130: Population Projections of the United States by Sex, Race, and Hispanic Origin, 1995 to 2050. Washington, DC: U.S. Bureau of the Census.

U.S. Department of Health and Human Services. 1998a. The President's Initiative on Race, Health Care Rx: Access for All. Barriers to Health Care for Racial and Ethnic Minorities: Access, Workforce Diversity, and Cultural Competence. Washington, DC: U.S. Department of Health and Human Services.

_. 1998b. Tobacco Use among U.S. Racial/Ethnic Minority Groups-African Americans, American Indians and Alaska Natives, Asian Americans and Pacific Islanders, and Hispanics: A Report of the Surgeon General. In Tobacco Control and Education Efforts. Atlanta, GA: U.S. Department of Health and Human Services, Center for Disease Control and Prevention, National Center for Chronic Disease Prevention and Health Promotion, Office on Smoking and Health. 
1999. Eliminating Racial and Ethnic Disparities in Health. Washington, DC: U.S. Department of Health and Human Services.

U.S. Department of Health and Human Services Goal 6 Workgroup. 1998. Cultural and Linguistic Competency as a Consumer Protection Issue in DHHS. Washington, DC: U.S. Department of Health and Human Services.

U.S. Department of Health and Human Services Office for Civil Rights. 1998. Title VI Prohibition against National Origin Discrimination-Persons with Limited-English Proficiency. Washington, DC: U.S. Department of Health and Human Services.

U.S. Department of Health and Human Services Office of the Secretary. 1999. Call for Comments on Draft Standards on Culturally and Linguistically Appropriate Health Care and Announcement of Regional Informational Meetings on Draft Standards. Federal Register, 15 December 1999, 64 (240): 70042-4.

Uba, L. 1992. Cultural Barriers to Health Care for Southeast Asian Refugees. Public Health Reports 107 (5): 544-8.

van Ryn, M., and J. Burke. 2000. The Effect of Patient Race and Socio-Economic Status on Physicians' Perceptions of Patients. Social Science E Medicine 50:813-28.

Wagner, T. H., and S. Guendelman. 2000. Healthcare Utilization among Hispanics: Findings from the 1994 Minority Health Survey. American Journal of Managed Care 6:355-64.

Weber, B. E., and B. M. Reilly. 1997. Enhancing Mammography Use in the Inner City: A Randomized Trial of Intensive Case Management. Archives of Internal Medicine 157 (20): 2345-9.

Weissman, J. S., R. Stern, S. L. Fielding, and A. M. Epstein. 1991. Delayed Access to Health Care: Risk Factors, Reasons, and Consequences. Annals of Internal Medicine 114 (4): 325-31.

Wenneker, M. B., and A. M. Epstein. 1989. Racial Inequalities in the Use of Procedures for Patients with Ischemic Heart Disease in Massachusetts. Journal of the American Medical Association 261 (2): 253-7.

Whittle, J., J. Conigliaro, C. B. Good, and M. Joswiak. 1997. Do Patient Preferences Contribute to Racial Differences in Cardiovascular Procedure Use? Journal of General Internal Medicine 12 (5): 267-73.

Williams, B. R. 1997. HPHC's Diversity Journey. In Harvard Pilgrim Health Care Diversity Journal, edited by B. Stern. Brookline, MA: Harvard Pilgrim Health Care.

Williams, D. R. 1999. Race, Socioeconomic Status, and Health: The Added Effects of Racism and Discrimination. Annals of the New York Academy of Sciences 896:173-88.

Witmer, A., S. D. Seifer, L. Finocchio, J. Leslie, and E. H. O’ Neil. 1995. Community Health Workers: Integral Members of the Health Care Work Force. American Journal of Public Health 85 (8): 1055-8.

Woloshin, S., N. A. Bickell, L. M. Schwartz, F. Gany, and H. G. Welch. 1995. Language Barriers in Medicine in the United States. Journal of the American Medical Association 273 (9): 724-8.

Woloshin, S., L. M. Schwartz, S. J. Katz, and H. G. Welch. 1997. Is Language a Barrier to the Use of Preventive Services? Journal of General Internal Medicine 12 (8): 472-7. 
Wood, D., N. Halfon, C. Donald-Sherbourne, R. M. Mazel, M. Schuster, J. S. Hamlin, M. Pereyra, P. Camp, M. Grabowsky, and N. Duan. 1998. Increasing Immunization Rates among Inner-City, African American Children: A Randomized Trial of Case Management. Journal of the American Medical Association 279 (1): 29-34.

Wright, A. L., A. Naylor, R. Wester, M. Bauer, and E. Sutcliffe. 1997. Using Cultural Knowledge in Health Promotion: Breastfeeding among the Navajo. Health Education and Behavior 24 (5): 625-39.

Xuo, D., and M. J. Fagan. 1999. Satisfaction with Methods of Spanish Interpretation in an Ambulatory Care Clinic. Journal of General Internal Medicine 14 (9): 547-50.

Zablocki, E. 1998. Health Plans Strive for Diversity. Healthplan 39 (2): 21-4.

*PLEASE CITE IN TEXT OR DELETE REFERENCE* Zubek, E. M. 1994. Traditional Native Healing. Alternative or Adjunct to Modern Medicine? Canadian Family Physician 40: 1923-31.

Zuckerman, M. J., L. G. Guerra, D. A. Drossman, J. A. Foland, and G. G. Gregory. 1996. Health-Care-Seeking Behaviors Related to Bowel Complaints: Hispanics versus Non-Hispanic Whites. Digestive Diseases and Sciences 41 (1): 77-82. 